\title{
KANSEI Information Processing of Human Body Movement
}

\author{
Mamiko Sakata $^{1}$ and Kozaburo Hachimura ${ }^{2}$ \\ ${ }^{1}$ Faculty of Culture and Information Science, Doshisha University \\ Tatara Miyakodani 1-3 Kyotanabe, Kyoto, Japan \\ msakata@mail.doshisha.ac.jp \\ ${ }^{2}$ College of Information Science and Engineering, Ritsumeikan University \\ Noji Higashi 1-1-1 Kusatsu, Shiga, Japan
}

\begin{abstract}
To quantitatively clarify the cognitive mechanism of KANSEI information conveyed by body movement, we conducted evaluation experiments and movement analysis by optical motion capture. We attempted to develop multiple regression models to investigate the effect of physical movement on subjective evaluation. The results show the relation between subjective feature of movements and the physical measurement. Thus, we expect that our research is effective to the guidance of dancing and acting, because it is possible to paraphrase sensitive image (KANSEI information) in another word (movement feature) which has strong relation with physical movement. Furthermore, this relational model which can replace human sensibility by digital data is useful for human-computer interaction.
\end{abstract}

Keywords: Body movement, KANSEI information, Motion capture.

\section{Introduction}

In the field of non-verbal communication research, the facial expression has been focused on, because it was considered that the body movement played only a secondary role(Ekman \& Friesen, 1975). However, in the research by authors, people can perceive KANSEI information(emotion, impression and image etc.) from body movement, even without facial expressions, and the body does not only have additional or secondary function, but also plays an important role as independent media in human communications(Sakata et al., 2004). Recently, there are some researches aiming to find the relation between KANSEI information and body movement, we don't very often find the researches with a quantitative underpinning. In effective communications among humans, and also between human and robots, to quantitatively examine KANSEI information conveyed by body movement is very important.

So far, it was tried to demonstrate the relationship between body motion and the impression arising from the motion. When doing this they extracted the physical values of movement through analysis of silhouette images from video image taken 
from directly in front of the performer (Camurri et al., 1999, 2002, Inoue et al., 2005). However, this method discards the three dimensional information.

Therefore, we use optical motion capture system to extract the characteristics of body movement, because a time series 3-D coordinates value are obtained.

In this paper, we aim to analyse the relation between the subjective evaluation of body movement and the physical measurement, and to quantitatively clarify the cognitive mechanism of KANSEI information conveyed by body movement.

\section{Target Movements}

In this research, Matsumoto's 7 Motives (Matsumoto, 1985) was subjected to study. It empirically verified the connection between images and movements. The 7 Motives are the seven dance movements that Matsumoto set up to be the minimum unit of expressions, in which each Motive contains clearly distinguishable emotional value. Matsumoto added the arms raising/lowering and closing/opening movements to the ordinary walking movement, and then set up the movements that involve the entire body, including exhalation and inhalation, as the "basic pattern" and called it the Natural Mv. Based on the Natural Mv., she varied the time, energy and design factors of movements, while retaining the characteristics of the Natural Mv. to the extent possible, and created three movements at both extremes. She named them as Happy Mv., Lonely Mv., Sharp Mv., Solemn Mv., Dynamic Mv., and Flowing Mv. Mv. is the abbreviation of "Motive" used by Matsumoto (see Table 1).

In the two experiments (evaluation experiment and motion capture), a skilled female dancer who is a 26-year-old majoring in dance with 15 years of training in modern dance, classical ballet, and contemporary dance performed 7 Motives. Before starting the experiments, she was briefed on the experiment, which was followed by

Table 1. Target Movement; Matsumoto's 7 Motives

\begin{tabular}{|c|c|c|c|c|}
\hline & Time & Energy & Design & Movement \\
\hline $\begin{array}{c}\text { Happy } \\
\text { Mv. }\end{array}$ & $\begin{array}{c}\text { M.M. } J=120 \\
\text { D.D.D.d.d }\end{array}$ & High & Skip Turn & \\
\hline Sharp Mv. & $\begin{array}{l}\text { M.M. } J=100 \\
\text { Adodod.Ad }\end{array}$ & High & Straight & \\
\hline $\begin{array}{c}\text { Lonely } \\
\text { Mv. }\end{array}$ & $\begin{array}{l}\text { M.M. J =36 } \\
\circ \text { 。 }\end{array}$ & Low & $\begin{array}{l}\text { Up \& Down, } \\
\text { Asymmetrical }\end{array}$ & \\
\hline $\begin{array}{c}\text { Natural } \\
\text { Mv. }\end{array}$ & $\begin{array}{c}\text { M.M. J }=96 \\
\text { 」d.d.d. }\end{array}$ & Normal & $\begin{array}{c}\text { Symmetrical, } \\
\text { Balance }\end{array}$ & \\
\hline $\begin{array}{c}\text { Flowing } \\
\text { Mv. }\end{array}$ & $\begin{array}{c}\text { M.M. J }=92 \\
\text { _...... }\end{array}$ & Normal & $\begin{array}{c}\text { Smooth } \\
\text {-faced turn }\end{array}$ & \\
\hline $\begin{array}{c}\text { Dynamic } \\
\text { Mv. }\end{array}$ & $\begin{array}{l}\text { M.M. J }=192 \\
\text { •dd.」d.d }\end{array}$ & High & Jump & \\
\hline $\begin{array}{l}\text { Solemn } \\
\text { Mv. }\end{array}$ & $\begin{array}{l}\text { M.M. J }=15 \\
\text {.dd }\end{array}$ & Low & $\begin{array}{c}\text { Symmetrical, } \\
\text { Balance }\end{array}$ & \\
\hline
\end{tabular}


several weeks rehearsal. During the rehearsal, the authors familiar with the 7 Motives were present and the performer rehearsed until she was able to represent the 7 Motives in a loyal manner.

\section{Extraction of KANSEI Information from Body Movement}

In this chapter, to extract KANSEI information from the body movement, we conducted an evaluation experiment using 7 Motives as the stimulus. We researched what the kinds of KANSEI information perceived from body movements and how the raters catch the feature of movement, and developed relation models to investigate the effect of movement feature on KANSEI information conveyed through the body movement.

Forty-eight raters (39 men, 9women, with average age of 22.4, $\mathrm{SD}=2.05$ ) participated in this experiment. To allow the raters to sufficiently observe the stimuli, movement image were projected on a large display.

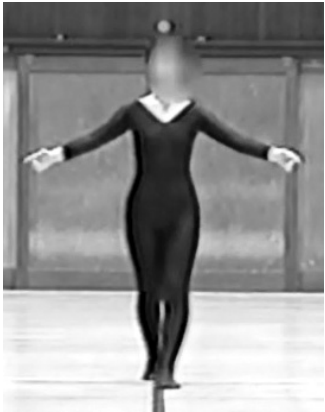

Fig. 1. Mosaic

Matsumoto's 7 Motives were newly acted out by the foregoing dancer and was recorded on a video-tape, which was used as the displayed stimulus. The face of the dancer in the image was obscured by mosaic to exclude the influence of the expression (see Figure 1). The name of each motive was not taught the raters.

After the raters were asked to glance over the two lists of rating terms, they were shown the display stimulus showing the motives one by one. After the same motive was shown, the vision changed to an interval. During the interval, the raters were asked to fill in the answer sheets with the following questions.

i. How much of the seven KANSEI information (happy, sharp, lonely, natural, flowing, dynamic and solemn) were expressed in each Motive: (Rated in the 5level scales ranging from 1.'not expressed' through 5. 'very clearly expressed')

ii. What kind of motion each motive showed: (Rated in the 5-level extreme scales by semantic differential method, see Table 2) This rating terms are selected in terms of time, energy and design; the elements that composes movement (Matsumoto, 1968).

Table 2. The Rating Terms for Movement Feature

\begin{tabular}{cl}
\hline \multirow{3}{*}{ Time } & T1: Speedy-Slow \\
& T2: Accentual-Smooth \\
& T3:Irregular-Regular \\
\hline \multirow{2}{*}{ Energy } & E1: Strong-Weak \\
& E2: Heavy-Light \\
& E3: Variable-Constant \\
\hline \multirow{2}{*}{ Design } & D1: Linear-Curved \\
& D2: Expansive-Shrinking \\
& D3: Balanced-Imbalance \\
\hline
\end{tabular}




\subsection{The Subjective Features of Body Movement}

\section{Rating on KANSEI Information of the 7 Motives}

The rating results using the 5-level scales for the seven KANSEI information (happy, sharp, lonely, natural, flowing, dynamic and solemn) were converted into numerical values (from 1 to 5 points). The mean value were obtained and tabulated in Table 3.

Table 3. Mean Value of KANSEI Information

\begin{tabular}{|c|c|c|c|c|c|c|c|c|}
\hline & \multicolumn{7}{|c|}{7 Motives } \\
\hline & & $\begin{array}{c}\text { Happy } \\
M_{V .}\end{array}$ & $\begin{array}{c}\text { Sharp } \\
M_{V} .\end{array}$ & $\begin{array}{l}\text { Lonely } \\
\text { Mv. }\end{array}$ & $\begin{array}{c}\text { Natural } \\
\text { Mv. }\end{array}$ & $\begin{array}{c}\text { Flowing } \\
M_{V} .\end{array}$ & $\begin{array}{c}\text { Dynami } \\
c M_{V} .\end{array}$ & $\begin{array}{c}\text { Solemn } \\
M v .\end{array}$ \\
\hline \multirow{7}{*}{ 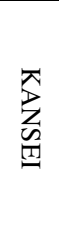 } & Happy & 4.56 & 1.98 & 1.79 & 2.19 & 4.08 & 4.33 & 1.58 \\
\hline & Sharp & 2.04 & 4.67 & 1.40 & 1.60 & 1.81 & 2.92 & 1.54 \\
\hline & Lonely & 1.29 & 2.25 & 3.81 & 2.54 & 1.73 & 1.40 & 3.67 \\
\hline & Natural & 1.94 & 1.56 & 2.75 & 3.27 & 2.42 & 1.71 & 2.54 \\
\hline & Flowing & 4.06 & 1.75 & 3.35 & 4.02 & 4.56 & 3.54 & 3.23 \\
\hline & Dynamic & 4.33 & 3.06 & 1.81 & 1.96 & 3.77 & 4.83 & 1.85 \\
\hline & Solemn & 1.43 & 3.63 & 2.96 & 2.25 & 1.85 & 1.65 & 3.90 \\
\hline
\end{tabular}

\section{Rating on Movement Features of the 7 Motives}

Table 4 shows the results of rating on movement features of the 7 Motives. "It applies" was made a score as one point to the left term of Table 2, and "It applies" was made a score as five points to the right term, and "There is not both" was made a score as three points.

Table 4. Mean Value of Movement Features

\begin{tabular}{rrrrrrrr}
\hline & $\begin{array}{c}\text { Happy } \\
\text { Mv. }\end{array}$ & Sharp Mv. & $\begin{array}{c}\text { Lonely } \\
\text { Mv. }\end{array}$ & $\begin{array}{c}\text { Natural } \\
\text { Mv. }\end{array}$ & $\begin{array}{c}\text { Flowing } \\
M_{V} .\end{array}$ & $\begin{array}{c}\text { Dynamic } \\
M_{V} .\end{array}$ & $\begin{array}{c}\text { Solemn } \\
M_{V .}\end{array}$ \\
\hline T1 & 1.77 & 2.19 & 4.85 & 3.88 & 3.17 & 1.25 & 4.81 \\
T2 & 2.88 & 1.25 & 4.63 & 4.08 & 3.79 & 1.81 & 4.42 \\
T3 & 3.75 & 4.50 & 3.06 & 4.54 & 3.63 & 3.40 & 3.98 \\
\hline E1 & 2.58 & 1.42 & 3.69 & 3.33 & 2.75 & 2.08 & 2.96 \\
E2 & 4.33 & 2.42 & 2.52 & 3.27 & 3.98 & 4.13 & 1.88 \\
E3 & 3.50 & 2.15 & 3.98 & 4.25 & 3.52 & 2.31 & 4.25 \\
\hline D1 & 3.85 & 1.38 & 4.60 & 3.15 & 4.52 & 2.21 & 3.85 \\
D2 & 2.58 & 2.75 & 2.79 & 2.79 & 2.04 & 1.69 & 2.69 \\
D3 & 2.58 & 2.17 & 3.00 & 1.77 & 2.48 & 2.67 & 2.17 \\
\hline
\end{tabular}

\subsection{The Relation Between KANSEI Information and Movement Feature}

In order to examine the relation between the perceived KANSEI Information and movement feature, we conducted multiple regression analyses using the nine evaluated scores of each movement feature (mean value of Table 4) as explanation variables, and the evaluated scores of the seven KANSEI Information (mean value of Table 3) as destination variables (by stepwise way). Table 5 shows the standardized coefficients $(\mathrm{p}<0.05)$ and the scores of adjusted $\mathrm{R}^{2}$. 
As shown by Table 5, a regression model with high significance was obtained for all of the KANSEI information, except for "flowing" (significance level p<0.05). In the author's earlier study, a multiple regression model for the flowing impression could not be obtained, too. From the values of the coefficient concerned with each model, we can find that "light" motion contribute to happy impression, while it is "strong" motion for sharp, "heavy" and "slow" for lonely, "balanced" and "weak" motion for natural, "speedy", "curved", "Expanding" and "heavy" motion for dynamic, and "heavy", "strong" motion for solemn. It seems that KANSEI information perceived from each motive are greatly affected by the energy factor of movement.

The relation between KANSEI information and movement feature is shown in the Figure 2.

Table 5. Relation between KANSEI Information and Movement Feature

\begin{tabular}{|c|c|c|c|c|c|c|c|}
\hline & $\mathrm{T} 1$ & $\mathrm{~T} 2$ & $\mathrm{~T} 3$ & D3 & E1 & E2 & adjusted $\mathrm{R}^{2}$ \\
\hline Happy $^{* *}$ & - & - & - & - & - & $0.952^{* *}$ & 0.887 \\
\hline Sharp $^{* *}$ & - & - & - & - & $-0.927^{* *}$ & - & 0.831 \\
\hline Lonely $^{* *}$ & $0.590^{*}$ & - & - & - & - & $-0.466^{*}$ & 0.924 \\
\hline Natural $^{* *}$ & - & - & - & $-0.427^{* * *}$ & $0.946^{* * *}$ & - & 0.952 \\
\hline Flowing & - & - & - & - & - & - & - \\
\hline Dynamic $^{* *}$ & $-1.178^{* *}$ & $0.468^{* *}$ & $-0.287^{* *}$ & - & - & $-0.213^{*}$ & 0.998 \\
\hline Solemn ${ }^{* * *}$ & - & - & - & - & $-0.180^{*}$ & $-1.006^{* * * *}$ & 0.991 \\
\hline
\end{tabular}

$<$ Movement Feature> $>$ <ANSEI $>$ <Movement Feature $>$

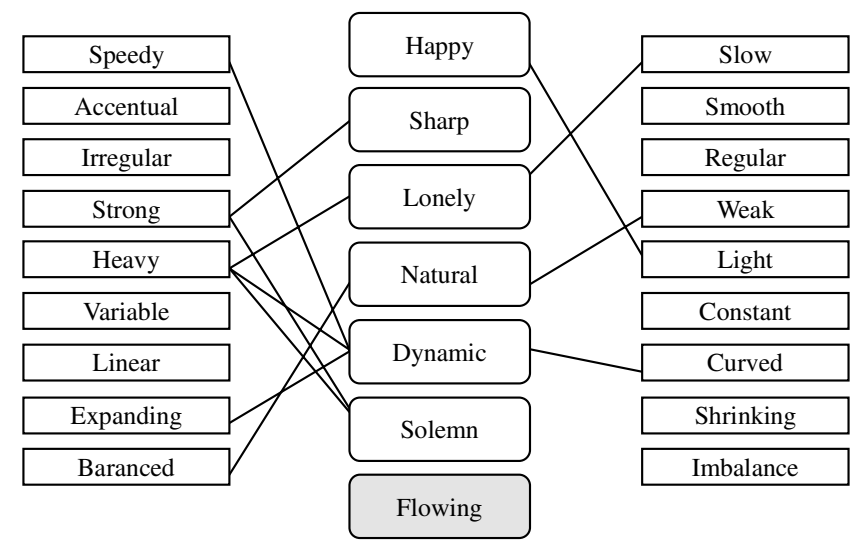

Fig. 2. Relation between the KANSEI Information and the Movement Feature

\section{Measurement of Body Movements}

In the preceding chapter, we examined the relation between KANSEI information perceived from body and the movement feature. It was clarified that KANSEI 
information perceived from each motive were greatly affected by the energy factor of movement. However, the feature of movement is only a subjective factor. It is necessary to examine the relation to the above-mentioned movement feature by the rater and a physical actual one. Therefore, we measured the dancer's movements of 7 Motives by using the optical motion capture.

\subsection{Optical Motion Capture}

The optical motion capture system employs video camera to track the motion of reflective markers attached to joints of the actor's body. The system uses LED's mounted around the camera lens. In this system, the centre of the marker images are matched from the various camera views using triangulation to compute their frame-to-frame positions in 3D space. Several problems often occur during the tracking process, including of markers, noisy or missing data and false reflections.

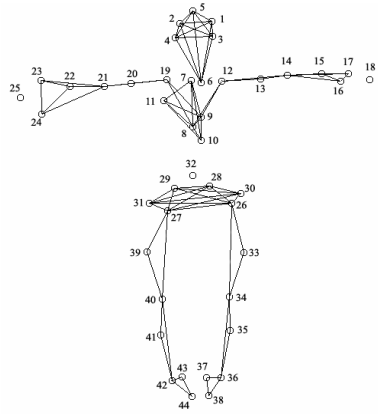

Fig. 3. Positions of Markers

In this experiment, 44 markers were put on the same dancer's body to the way of Figure 3, and the movement was measured with ten LED's cameras. The acquired data is obtained as a time series coordinates value( $\mathrm{x}, \mathrm{y}$, z) of each marker position by each frame (frame rate $1 / 60 \mathrm{sec}$.).

\subsection{Physical Measurement}

When the motion capture is used, the enormous quantity of data by each part is obtained. Then, it becomes important to discover a significant parameter which influences KANSEI information.

In this paper, the following 16 measurements are calculated as the physical parameters that seem to influence KANSEI information; mean velocity of hand, SD velocity of hand, mean acceleration of hand, SD acceleration of hand, Mean velocity

$<$ Physical Measurements $>$

P1: Mean velocity of hand

P2: SD velocity of hand

P3: Mean acceleration of hand

P4: SD acceleration of hand

P5: Mean velocity of foot

P6: SD velocity of foot

P7: Mean acceleration of foot

P8: SD acceleration of foot

P9: Mean velocity of root

P10: SD velocity of root

P11: Mean acceleration of root

P12: SD acceleration of root

P13: Mean dimensions of opened body

P14: SD dimensions of opened body

P15: Mean height of root

P16: SD height of root

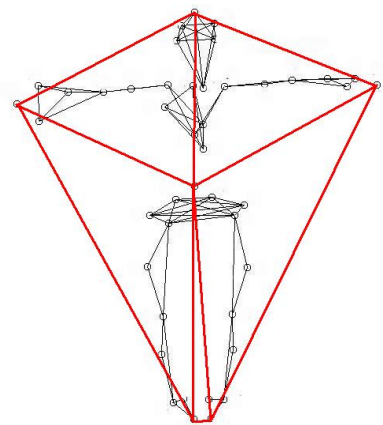

Fig. 4. Dimensions of Opened Body 
of foot, SD velocity of foot, Mean acceleration of foot, SD acceleration of foot, Mean velocity of root, SD velocity of root, Mean acceleration of root, SD acceleration of root, Mean dimensions of opened body, SD dimensions of opened body, mean height of root, SD height of root. Because 7 Motives are basically symmetric movements, only the right were adopted as for measurements of both hands and feet. The dimensions of opened body are the sum total of the triangular area made when drawing a straight line from five end parts (head top, both hands, both ankles) to the root (Figure 4).The height of root was based on the absolute origin of the capturing area.

\subsection{Result of Physical Measurements}

Table 7 shows the mean value and standard deviation of physical measurement of each motive. In the next chapter, we examine the relation between this result and the subjective evaluation by the raters.

Table 7. Result of Physical Measurement.

\begin{tabular}{|c|c|c|c|c|c|c|c|c|}
\hline & & $\begin{array}{c}\text { Happy } \\
M_{v} .\end{array}$ & $\begin{array}{c}\text { Sharp } \\
M v .\end{array}$ & $\begin{array}{c}\text { Lonely } \\
M V .\end{array}$ & $\begin{array}{c}\text { Natural } \\
M v .\end{array}$ & $\begin{array}{c}\text { Flowin } \\
g M_{v} .\end{array}$ & $\begin{array}{c}\text { Dynami } \\
c M v .\end{array}$ & $\begin{array}{c}\text { Solemn } \\
M_{v} .\end{array}$ \\
\hline P1 & \multirow{2}{*}{$(\mathrm{cm} / \mathrm{sec})$} & 40.82 & 26.04 & 5.13 & 7.27 & 34.49 & 51.16 & 3.34 \\
\hline $\mathrm{P} 2$ & & 19.32 & 21.89 & 3.66 & 3.28 & 17.40 & 24.01 & 2.42 \\
\hline P3 & \multirow{2}{*}{$\left(\mathrm{cm} / \mathrm{sec}^{2}\right)$} & 6.94 & 5.00 & 1.56 & 1.70 & 4.66 & 9.97 & 1.26 \\
\hline P4 & & 5.63 & 3.94 & 2.16 & 1.74 & 4.52 & 7.46 & 1.98 \\
\hline P5 & \multirow{2}{*}{$(\mathrm{cm} / \mathrm{sec})$} & 24.61 & 22.57 & 2.76 & 15.57 & 17.05 & 47.96 & 2.47 \\
\hline P6 & & 14.89 & 24.17 & 3.83 & 7.72 & 10.31 & 22.98 & 4.29 \\
\hline P7 & \multirow{2}{*}{$\left(\mathrm{cm} / \mathrm{sec}^{2}\right)$} & 7.52 & 4.86 & 1.07 & 2.11 & 3.00 & 10.50 & 0.55 \\
\hline P8 & & 8.29 & 5.64 & 1.85 & 1.66 & 2.31 & 8.97 & 0.68 \\
\hline P9 & \multirow{2}{*}{$(\mathrm{cm} / \mathrm{sec})$} & 19.35 & 11.29 & 1.84 & 13.41 & 10.46 & 44.54 & 1.89 \\
\hline $\mathrm{P} 10$ & & 7.68 & 6.13 & 1.35 & 2.31 & 5.28 & 7.90 & 2.74 \\
\hline P11 & \multirow{2}{*}{$\left(\mathrm{cm} / \mathrm{sec}^{2}\right)$} & 3.58 & 1.11 & 0.40 & 0.66 & 1.00 & 3.69 & 0.29 \\
\hline $\mathrm{P} 12$ & & 3.57 & 0.98 & 0.65 & 0.36 & 0.62 & 2.29 & 0.33 \\
\hline $\mathrm{P} 13$ & \multirow{2}{*}{$\left(\mathrm{cm}^{2}\right)$} & 7579.24 & 7146.98 & 6315.32 & 7641.95 & 8030.88 & 9277.37 & 8464.56 \\
\hline P14 & & 2867.52 & 3425.65 & 2061.86 & 2554.98 & 2442.39 & 2547.81 & 2799.85 \\
\hline P15 & \multirow{2}{*}{$(\mathrm{cm})$} & 1.44 & -0.92 & -13.24 & -2.04 & -1.08 & 7.20 & -10.88 \\
\hline P16 & & 6.19 & 5.64 & 13.40 & 0.77 & 8.93 & 13.98 & 9.31 \\
\hline
\end{tabular}

\section{Relation Between Subjective Evaluation and Physical Measurement}

Section 3.5 above, we attempted to develop a multiple regression model of the seven KANSEI information using movement features. However, both of them are only subjective evaluation. In this chapter, we made sure the relation between the subjective evaluation by the rater and the physical movement by motion capture.

Table 8 shows the correlation matrix of subjective evaluation and physical measurements. Many of physical measurements correlated with "speed-slow", because P1 P12 were velocity and acceleration. All of them correlated with at least one subjective term except for $<$ mean dimensions of opened body $>$.

Next, we conducted multiple regression analysis using the nine evaluated scores of each movements features (Table 4) as explanation variables, and the physical 
measurement (Table 7) as destination variables (by stepwise way). Table 9 shows standardized coefficients $(\mathrm{p}<0.05)$ and the scores of adjusted R2.

As the result, we obtained a multiple regression model for all subjective features (significance level $\mathrm{p}<0.05$ ). When each feature was observed, the physical measurement that contributes to "Speedy-Slow" is $<$ SD velocity of root $>$. Similarly, $<$ mean and SD velocity of foot $>$ contribute to "Accentual-Smooth", whereas $<$ SD height of root $>$ is the factors of "Irregular-Regular". $<$ SD velocity of foot $>$ contributes for "Strong-Weak", whereas it was <mean velocity of hand $>$ for "HeavyLight" and "Variable-Constant". <SD velocity of hand and foot $>$ contribute for "Linear-Curved", and $<$ SD acceleration of hand and foot $>,<$ mean velocity of root $>$ are the factors of "Expanding-Shrinking". <Mean dimensions of the opened body $>$

Table 8. Correlation Matrix of Subjective Evaluation and Physical Measurements

\begin{tabular}{|c|c|c|c|c|c|c|c|c|c|c|}
\hline & \multicolumn{9}{|c|}{ Movement Features } \\
\hline & & $\mathrm{T} 1$ & $\mathrm{~T} 2$ & $\mathrm{~T} 3$ & E1 & E2 & E3 & D1 & D2 & D3 \\
\hline \multirow{16}{*}{ 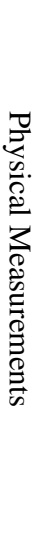 } & P1 & $-0.932^{* *}$ & -0.711 & -0.229 & -0.633 & $0.811^{*}$ & -0.718 & -0.325 & $-0.783^{*}$ & 0.318 \\
\hline & P2 & $-0.941^{* *}$ & $-0.880^{* *}$ & -0.050 & $-0.839^{*}$ & 0.602 & $-0.889^{* *}$ & -0.532 & -0.626 & 0.230 \\
\hline & P3 & $-0.945^{* *}$ & $-0.773^{*}$ & -0.233 & -0.661 & 0.728 & $-0.775^{*}$ & -0.450 & $-0.764^{*}$ & 0.326 \\
\hline & P4 & $-0.908^{* *}$ & -0.706 & -0.323 & -0.614 & 0.749 & -0.729 & -0.341 & $-0.807^{*}$ & 0.400 \\
\hline & P5 & $-0.920^{* *}$ & $-0.787^{*}$ & -0.053 & -0.646 & 0.687 & $-0.767^{*}$ & -0.605 & -0.744 & 0.108 \\
\hline & P6 & $-0.909^{* *}$ & $-0.994^{* * *}$ & 0.201 & $-0.927^{* *}$ & 0.370 & $-0.961^{* *}$ & $-0.832^{*}$ & -0.449 & 0.003 \\
\hline & P7 & $-0.947^{* *}$ & $-0.781^{*}$ & -0.161 & -0.630 & 0.708 & -0.743 & -0.514 & -0.662 & 0.263 \\
\hline & P8 & $-0.939^{* *}$ & $-0.807^{*}$ & -0.140 & -0.656 & 0.636 & -0.749 & -0.515 & -0.493 & 0.309 \\
\hline & P9 & $-0.821^{*}$ & -0.640 & -0.161 & -0.475 & 0.687 & -0.626 & -0.497 & $-0.769^{*}$ & 0.152 \\
\hline & P10 & $-0.963^{* * *}$ & $-0.816^{*}$ & -0.025 & $-0.779^{*}$ & 0.675 & $-0.761^{*}$ & -0.469 & -0.619 & 0.166 \\
\hline & P11 & $-0.873^{*}$ & -0.606 & -0.251 & -0.454 & $0.776^{*}$ & -0.538 & -0.291 & -0.589 & 0.329 \\
\hline & P12 & $-0.764^{*}$ & -0.497 & -0.250 & -0.356 & 0.690 & -0.395 & -0.142 & -0.324 & 0.384 \\
\hline & P13 & 0.059 & 0.028 & -0.273 & -0.008 & -0.066 & -0.122 & -0.114 & -0.639 & 0.046 \\
\hline & P14 & -0.454 & -0.690 & 0.714 & $-0.810^{*}$ & -0.174 & -0.525 & -0.717 & 0.201 & -0.501 \\
\hline & P15 & $-0.932^{* *}$ & -0.750 & 0.154 & -0.647 & $0.777^{*}$ & -0.673 & -0.555 & -0.672 & -0.101 \\
\hline & P16 & -0.010 & 0.005 & $-0.878^{* *}$ & 0.034 & 0.003 & -0.231 & 0.206 & -0.518 & $0.838^{\circ}$ \\
\hline
\end{tabular}

Table 9. Relationship between subjective evaluation and physical Measurements

\begin{tabular}{|c|c|c|c|c|c|c|c|c|c|c|c|}
\hline & $\mathrm{P} 1$ & $\mathrm{P} 2$ & $\mathrm{P} 4$ & P5 & P6 & P8 & P9 & P10 & P13 & P16 & $\begin{array}{c}\text { adjusted } \\
\mathrm{R}^{2}\end{array}$ \\
\hline $\mathrm{T} 1^{* * *}$ & - & - & - & - & - & - & - & -0.963 & - & - & 0.913 \\
\hline $\mathrm{T} 2^{* * *}$ & - & - & - & 0.173 & -1.14 & - & - & - & - & - & 0.995 \\
\hline $\mathrm{T} 3^{* *}$ & - & - & - & - & - & - & - & - & - & -0.878 & 0.72 \\
\hline $\mathrm{E} 1^{* *}$ & - & - & - & - & -0.927 & - & - & - & - & - & 0.83 \\
\hline $\mathrm{E} 2^{*}$ & 0.811 & - & - & - & - & - & - & - & - & - & 0.588 \\
\hline $\mathrm{E} 3^{* *}$ & - & - & - & - & -0.961 & - & - & - & - & & 0.908 \\
\hline $\mathrm{D} 1^{* * *}$ & - & 1.285 & - & - & -2 & - & - & - & - & - & 0.972 \\
\hline $\mathrm{D} 2^{* * *}$ & - & - & -1.575 & - & - & 1.328 & -0.495 & - & - & - & 0.992 \\
\hline D3 $3^{* *}$ & - & - & - & - & - & - & - & - & -0.572 & 1.146 & 0.901 \\
\hline
\end{tabular}

${ }^{*} \ldots \mathrm{p}<0.05,{ }^{* *} \ldots \mathrm{p}<0.01,{ }^{* * *} \ldots \mathrm{p}<0.001 \quad \nVdash$ The table shows only statistically-meaningful items of physical feature. 
and $<$ SD height of root $>$ contribute for "Balanced-Imbalance". From these results, it is assumed that the feature of movement that the raters subjectively felt can be replaced with the physical measurement.

\section{Discussion}

When the results of this research are brought together, it is schematised as shown in the following Figure 5.

The left lines show the relation between KANSEI information and movement feature in the raters cognition. On the right side, the relation between subjective value (movement feature) and physical value are connected. Put simply, the subjective and the objective feature of movement conveyed KANSEI information was revealed. For example, lonely motion is slow and heavy, and it is the actual movement that the hand is slowly moved and the difference of fast and slow of root (waist) is not applied. This result is effective to the guidance of dancing and acting, because it is possible to paraphrase sensitive image (KANSEI information) in another word (movement feature) which has strong relation with physical movement.

The reason of the failure to develop a multiple regression model on "flowing" image is attributed to the fact that it is difficult to replace "flowing" with another word representing motion. It seems that "flowing" is not KANSEI information, but movement feature.

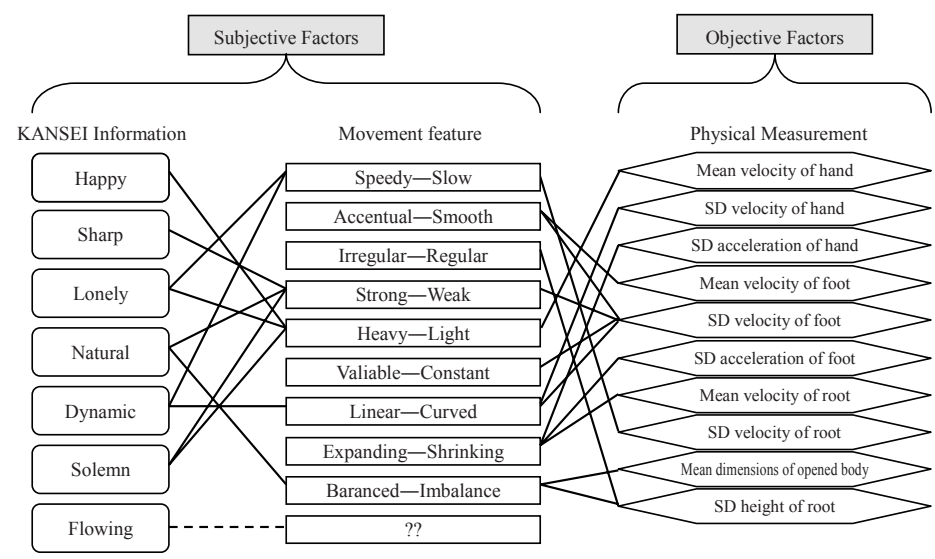

Fig. 5. Relation among KANSEI Information and Movement Feature and Physical Measurement

\section{Conclusion and Future Work}

To quantitatively clarify the cognitive mechanism of KANSEI information conveyed by body movement, we conducted evaluation experiments and movement analysis by optical motion capture. We attempted to develop multiple regression models to 
investigate the effect of physical movement on subjective evaluation. The results show the relation between subjective feature of movements and the physical measurement. Thus, we expect that our research is effective to the guidance of dancing and acting, because it is possible to paraphrase sensitive image (KANSEI information) in another word (movement feature) which has strong relation with physical movement. Furthermore, this relational model which can replace human sensibility by digital data is useful for human-computer interaction.

In the future, we will examine the all kinds of movement, not apply only to 7 Motives. Furthermore, we will verify the results of this paper and construct a more exquisite relational model that various movements are covered.

\section{References}

1. Camurri, A., Coletta, P., Mazzarino, B., Trocca, R., Volpe, G.: Improving the man-machine interface through the analysis of expressiveness in human movement. In: Proc. 2002 IEEE ROMAN conf., pp. 417-421 (2002)

2. Camurri, A., Hashimoto, S., Suzuki, K., Trocca, R.: KANSEI Analysis of Dance Performance. IEEE International Conference on System, Man and Cybernetics, vol. IV, pp. 327-332 (1999)

3. Ekman, P., Friesen, W.V.: Unmasking the face. Prentice-Hall, Inc., NJ (1975)

4. Inoue, M., Iwadate, Y., Shiba, M., Auzuki, M.: Kansei Feature Extraction Related to Human body-Movements. The Journal of The Virtual Reality Society of Japan 1(10), 31-40 (2005) (in Japanese)

5. Matsumoto, C.: Dance research: Problem situation and learning of problem solving II Qualities of movements and feeling values. Lecture at the Tenth Congress of the international Association of Physical Education and Sport for Girls and Women. Lerwick (1985)

6. Matsumoto, C.: An introduction of kinesiology. Taishukan Inc., Tokyo (1968)

7. Sakata, M., Shiba, M., Maiya, K., Tadenuma, M.: Human Body as the Medium in Dance Movement. International Journal of Human- Computer Interaction 17(3), 427-444 (2004) 